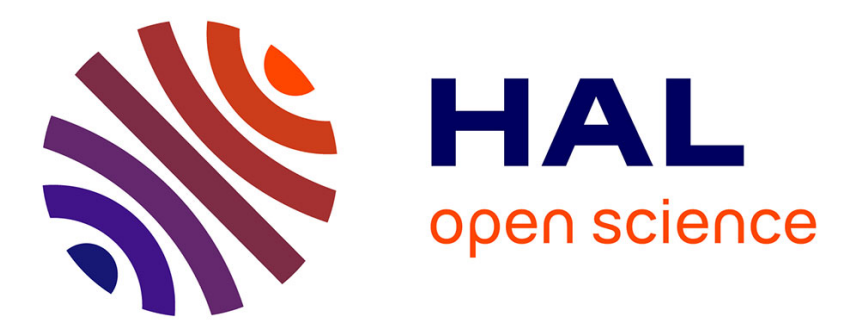

\title{
Sur la structure fine des triplets de l'hélium
}

\author{
J. Brochard, R. Chabbal, H. Chantrel, P. Jacquinot
}

\section{To cite this version:}

J. Brochard, R. Chabbal, H. Chantrel, P. Jacquinot. Sur la structure fine des triplets de l'hélium. J. Phys. Radium, 1952, 13 (10), pp.433-437. 10.1051/jphysrad:019520013010043300 . jpa-00234617

\section{HAL Id: jpa-00234617 https://hal.science/jpa-00234617}

Submitted on 1 Jan 1952

HAL is a multi-disciplinary open access archive for the deposit and dissemination of scientific research documents, whether they are published or not. The documents may come from teaching and research institutions in France or abroad, or from public or private research centers.
L'archive ouverte pluridisciplinaire HAL, est destinée au dépôt et à la diffusion de documents scientifiques de niveau recherche, publiés ou non, émanant des établissements d'enseignement et de recherche français ou étrangers, des laboratoires publics ou privés. 


\title{
LE JOURNAL DE PHYSIQUE
}

$\mathbf{E T}$

\section{LE RADIUM}

\section{SUR LA STRUGTURE FINE DES TRIPLETS DE L'HÉLIOM}

\author{
Par MM. J. BROCHARD, R. CHABBAL, H. CHANTREL et P. JACQUINOT. \\ Laboratoire Aimé-Cotton, C. N. R. S., Bellevue.
}

\begin{abstract}
Sommaire. - La structure fine de trois niveaux de l'hélium $\left(2^{3} P, 3^{3} P, 3^{3} D\right)$ a été mesurée à la température de l'hydrogène liquide. Les raies étaient enregistrées au moyen d'un interféromètre FabryPérot photoélectrique à enregistrement direct. Pour les niveaux $2^{3} \mathrm{P}$ et $3^{3} \mathrm{D}$, les mesures confirment celles de Hansen avec une précision accrue, permettant de relever certains écarts avec la meilleure théorie, qui est celle d'Araki. Pour le niveau $3^{\mathrm{a}} P$, il s'avère que les mesures considérées jusqu'à présent comme les meilleures étaient entachées d'une grosse erreur due à l'auto-absorption et c'est encore la théorie d'Araki qui rend le mieux compte de la structure observée.
\end{abstract}

Introduction. - Il est connu depuis longtemps que la structure, très serrée, des niveaux de triplets de l'hélium, • est anormale et ne peut pas s'interpréter par les modèles simples du couplage $L S$ ou du couplage $j-j$. Cette structure a fait l'objet d'un certain nombre de recherches expérimentales et théoriques. La question a été étudiée, pour la première fois, par Heisenberg [1]; ensuite, les travaux les plus importants sont ceux de Breit [2], puis ceux d'Araki [3] qui donnent la théorie la plus complète, la seule à laquelle nous nous référerons. Les divers résultats expérimentaux sont assez divergents, ce qui n'est pas étonnant étant donné que les raies sont très rapprochées et que leur largeur Doppler est relativement grande. Afin de réduire la largeur Doppler des mesures ont été faites par Hansen [4] en plongeant la source dans l'hydrogène liquide. Mais la température du gaz luminescent est toujours supérieure à celle du bain et cela d'autant plus que . la puissance électrique développée dans la lampe est plus grande et si l'on diminue trop cette puissance, les raies deviennent trop faibles pour être étudiées avec les dispositifs classiques à haute résolution. Or, l'interféromètre de Fabry-Perot photoélectrique à enregistrement dont le principe a été décrit antérieurement [5] permet d'étudier des raies très faibles et nous avons pensé qu'il permettrait d'obtenir des résultats plus sûrs que les précédents. On doit ainsi pouvoir travailler jusqu'à une température voisine de celle de l'héliùm liquide; les mesures présentées ici ont, cependant, été faites seulement avec de l'hydrogène liquide.
1. Conditions expérimentales. - 1. I. Source. - C'est la lampe à cathode creuse de Schüler qui nous a semblé la plus avantageuse en ce qui concerne le refroidissement, l'économie du gaz liquẻfié et la simplicité de construction; la figure I représente le modèle utilisé. Avec des pressions d'hélium de l'ordre de $0,5 \mathrm{~mm}$ de $\mathrm{Hg}$, la tension aux bornes était de l'ordre de $200 \mathrm{~V}$ et les intensités utilisées ont varié entre 6 et $0,3 \mathrm{~mA}$, ce qui correspond à des puissances comprises entre 1,2 et $0,06 \mathrm{~W}$. D'après les largeurs de raies observées, l'écart de température entre le gaz et le bain ne dépassait jamais quelques degrès.

1.2 Dispositif interférométrique. - Il suffira d'en rappeler très brièvement le principe : dans le plan focal de la lentille située derrière l'étalon Fabry-Pérot et sur l'axe de l'étalon, est placé un trou dont le diamètre est une faible fraction de celui du premier anneau : la géométrie du faisceau éclairant l'étalon est telle que toute l'étendue disponible après la sortie du monochromateur soit, après la sortie de l'étalon, définie par une partie de l'étalon et l'ouverture angulaire du trou : dans ces conditions, toute la lumière disponible sur une longueur d'onde $\lambda$ telle que $2 n e=p \lambda$ passe dans le trou et est reçue sur une cellule à multiplicateurs (refroidie à l'azote liquide) dont le courant, après adaptation des impédances par un "cathode-follower " est enregistré par un potentiomètre électronique Speedomax. On peut montrer qu'avec un tel dispositif on recueille beaucoup plus de lumière qu'avec tout autre système 
dispersif, interférentiel ou non, de même résolution. L'exploration des longueurs d'onde est effectuée par variation de la pression dans l'enceinte qui contient

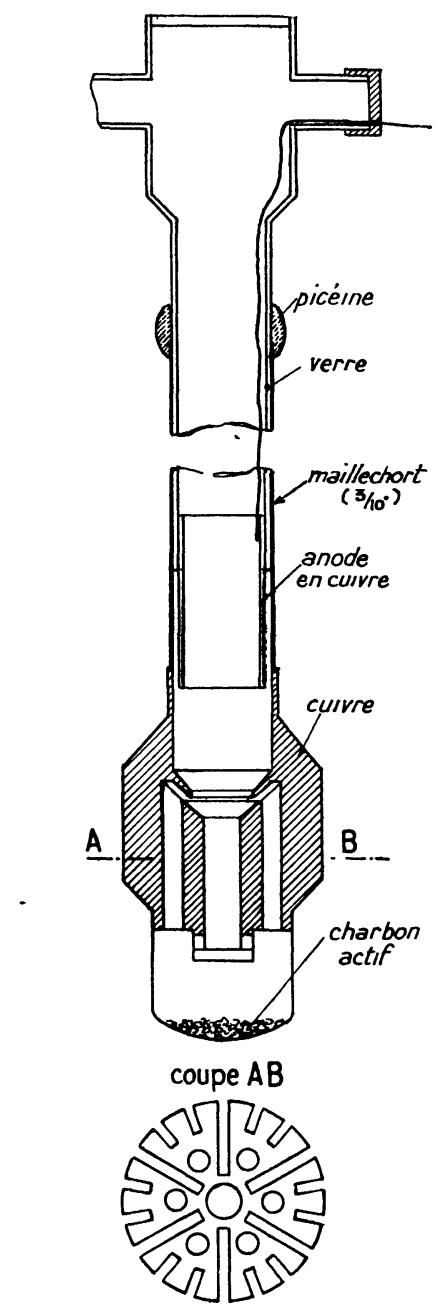

Fig. I. - Schémà de la lampe à cathode creuse.

l'étalon, ce qui fait ainsi varier $n$ : un dispositif simple rend cette variation de pression proportionnelle au déroulement du papier du Speedomax, ce qui permet d'obtenir un enregistrement avec une échelle linéaire en $\lambda$. Si l'ordre d'interférence est grand il peut être utile, afin d'avoir une échelle assez dilatée en $\lambda$, d'utiliser un gaz moins réfringent que l'air et nous avons, dans plusieurs cas, utilisé l'hélium ou un mélange d'hélium et d'air.

Les raies sont sélectionnées par un monochromateur placé avant l'ensemble interférométrique et dont la fente, largement ouverte, est conjuguée des lames de l'étalon; la surface utilisée sur les lames est de $7 \mathrm{~cm}^{2}$. La distance des lames est de l'ordre de $18 \mathrm{~mm}$ pour la plupart des raies.
2. Niveaux et raies étudiés. - La figure 2 indique les raies et les niveaux étudiés.

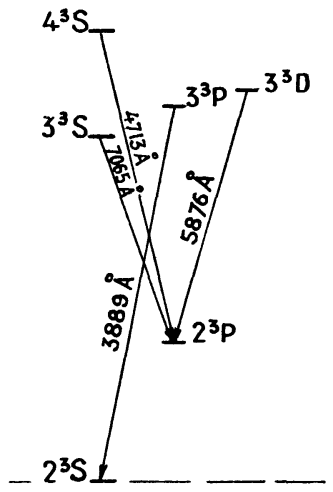

Fig. 2. - Transitions étudiées.

2. г. Niveau $2^{3} P$. - On peut l'atteindre par la raie $2^{3} P-3{ }^{3} S\left(7\right.$ o65 $\AA$ ) et par la raie ${ }^{3} P-4{ }^{3} S$ (4 7r $3 \AA$ ) : la première de ces raies a déjà été étudiée par Houston [6] (aịr liquide) et la deuxième par Hansen [4] (hydrogène liquide). Le tableau I donne les différents résultats et la décomposition de la raie, les notations étant indiquées sur la figure 3.

Dans l'ensemble, nos résultats pour ce niveau confirment assez bien les séparations des auteurs précédents. Quant aux intensités elles̉ sont, à la

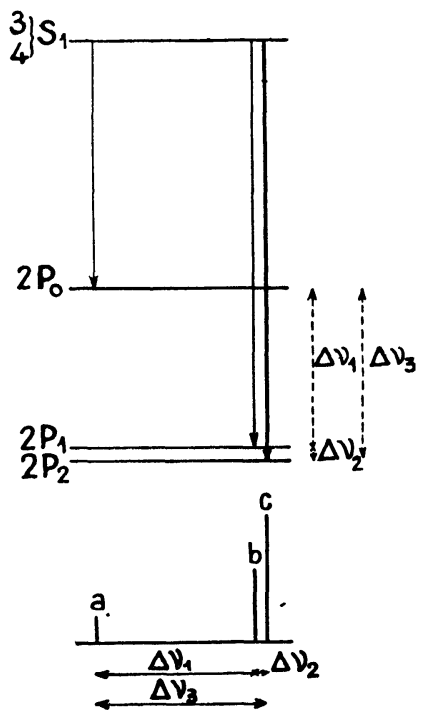

Fig. 3. - Structure fine des transitions $2{ }^{3} P-{ }^{3} S$.

précision des mesures, celles que donnerait un couplage Russell-Saunders.

Les figures 4 et 5 montrent un exemple d'enregistrements directs obtenus avec les raies 7065 et $47 \mathrm{r} 3 \AA$. 
Tableau I. - Niveau $2^{3} P$.

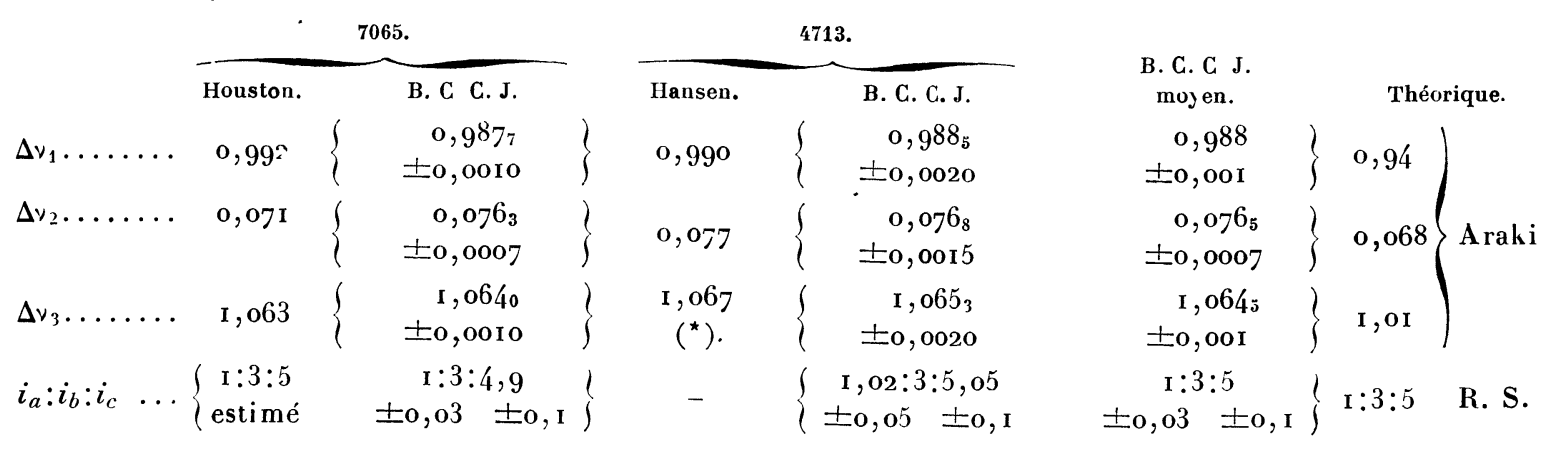

(*) Cette composante est probablement très légèrement auto-absorbée.

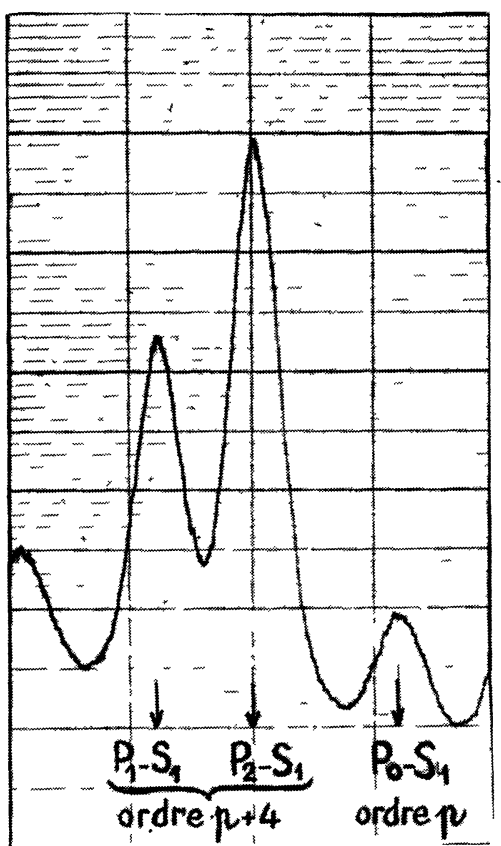

Fig. 4. - Enregistrement de la structure fine de la raie $7065 \AA\left(2^{3} P-3{ }^{8} S\right)$.

2.2. Niveau $3{ }^{3} P$. - Seule la raie ${ }^{3} S-3{ }^{3} P$ (3 $889 \AA$ ) permet d'étudier ce niveau : sa structure est indiquée sur la figure 6. Jusqu'ici, seuls Gibbs et Krüger [7] avaient obtenu la résolution en un triplet, avec une cathode creuse refroidie à l'air liquide. Le tableau II montre leurs résultats et les nôtres, qui sont tout à fait différents. En réalité, les séparations observées par Gibbs et Krüger sont dues uniquement au renversement de la raie. Nous avons vérifié que cette raie, dont le niveau inférieur est métastable, est très facilement renversée et nous avons pu facilement reproduire l'aspect observé

(1) L'inexactitude du résultat de Gibbs et Krüger a été aussi signalée par Bradley et Kuhn [8] lors d'un travail récent sur l'effet isotopique de l'hélium.

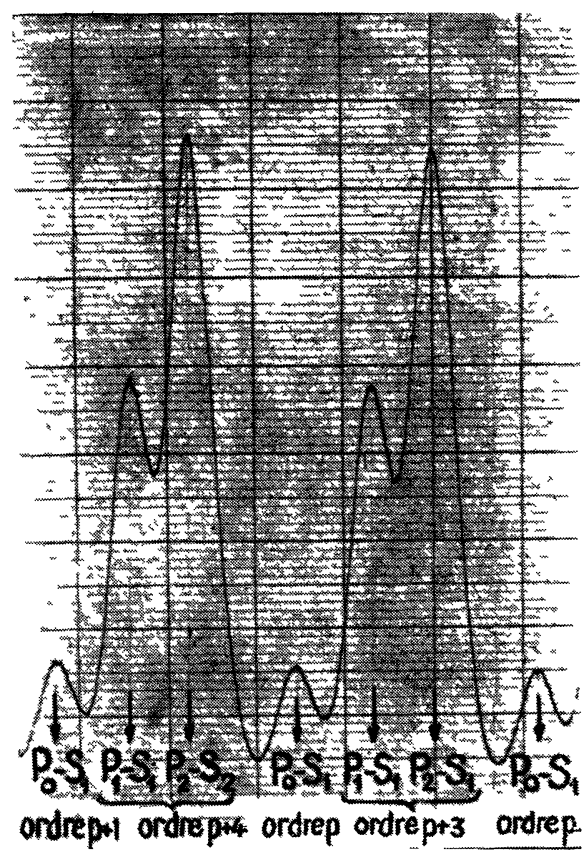

Fig. 5. - Enregistrement de la structure fine de la raie 47 r $3 \AA\left(2^{3} P-4{ }^{3} S\right)$.

Tableau II. - Niveau $3{ }^{3} P$.

$\left.\begin{array}{cccc}\text { Houston. } & \begin{array}{c}\text { Gubbs } \\ \text { et Kruger. }\end{array} & \left.\begin{array}{c}\text { B. C. C.J. } \\ 0,280 \\ \pm 0,010\end{array}\right\} & \text { Théorique } \\ 0,28_{6} & - & \left\{\begin{array}{c}0,025\left(^{\star \star}\right) \\ \pm 0,010\end{array}\right\} & 0,0176 \\ 0,02\left(^{\star}\right) & 0,165 & \left\{\begin{array}{c}0,265 \\ \pm 0,020\end{array}\right\} & 0,218 \\ 0,27\left(^{\star}\right) & 0,192 & \left\{\begin{array}{c}0,290 \\ \pm 0,015\end{array}\right. & 0,235\end{array}\right\}$ Araki

$\left(i_{b}+l_{c}\right): i_{a} \cdot\left\{\begin{array}{c}8: \mathrm{I} \\ \text { estimé }\end{array}\right\}(\mathrm{IO}+7): 3 \rightarrow 8: \mathrm{I} \quad 8: \mathrm{I} \quad$ R. S.

(*) Resultat obtenu par IIouston en admettant pour $\Delta v_{1}: \Delta v_{2}$ la même raleur que pour le niveau $2^{3} P$.

$\left({ }^{*}\right)$ estimé d'après la largeur de l'ensemble $b+c$. 
par Gibbs et Krüger. Ce renversement tend à disparaitre si l'on diminue fortement le courant dans la lampe; pour s'en affranchir, il est nécessaire d'aban-

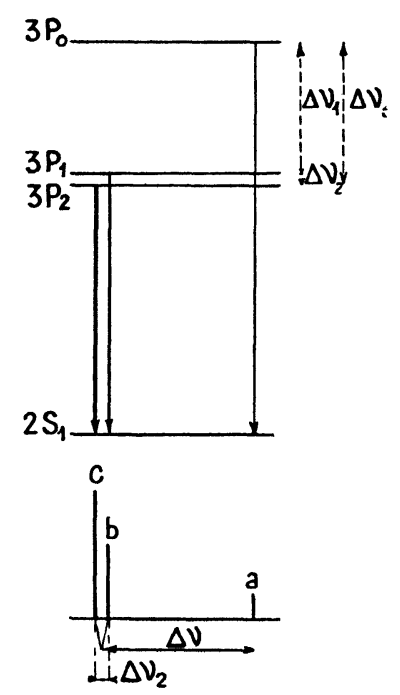

Fig. 6. - Structure fine de la transition $2{ }^{3} S-3{ }^{3} P$.

donner le régime de cathode creuse qui crée une inhomogénéité dans la décharge : pour $i=2 \mathrm{~mA}$ et $p=0,3 \mathrm{~mm}$ de $\mathrm{Hg}$, on obtient un régime beaucoup moins brillant et la raie apparait alors comme un doublet, les niveaux $P_{1}$ et $P_{2}$ étant presque confondus; cependant, l'ensemble, non résolu, des deux composantes $b$ et $c$ est encore un peu autoabsorbé, car le rapport des intensités $\frac{b+c}{a}$ croit quand on diminue l'intensité de courant et se rapproche du rapport théorique $L S$ mais sans l'atteindre tout à fait, même pour un courant aussi faible que $0,3 \mathrm{~mA}$. Pour ce très faible courant, l'élargissement par auto-absorption est certainement négli- geable et la largeur de $b+c$ est d'environ $0,020 \mathrm{~cm}^{-1}$ supérieure à celle de $a$ : c'est de cette mesure qu'est déduite l'évaluation de $\Delta \nu_{2}$. Donc, conformément à la théorie d'Araki, mais contrairement aux conclusions de Gibbs et Krüger, le niveau $3^{3} P$ est aussi mal décrit par le couplage $L S$ que le niveau ${ }_{2}^{3} P$.

2.3. Niveau $3{ }^{3} D$. - La raie $5876 \AA\left({ }^{3} P-3{ }^{3} D\right)$, qui permet d'étudier ce niveau, est, en principe,

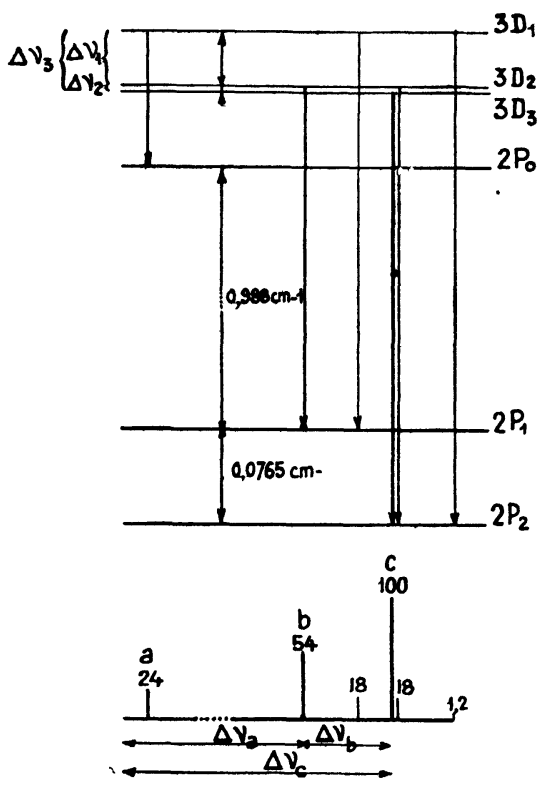

Fig. 7. - Structure fine de la transition $2^{3} P-3^{3} D$.

un sextuplet (fig. 7), mais que l'on ne peut, à la température de l'hydrogène liquide, résoudre qu'en trois composantes. Leurs distances et les écarts que l'on peut en déduire pour les niveaux $3^{3} D$, sont données sur le tableau III.

$$
\begin{aligned}
& \text { Tableau III. - Niveau } 3^{3} \mathrm{D} \text {. }
\end{aligned}
$$

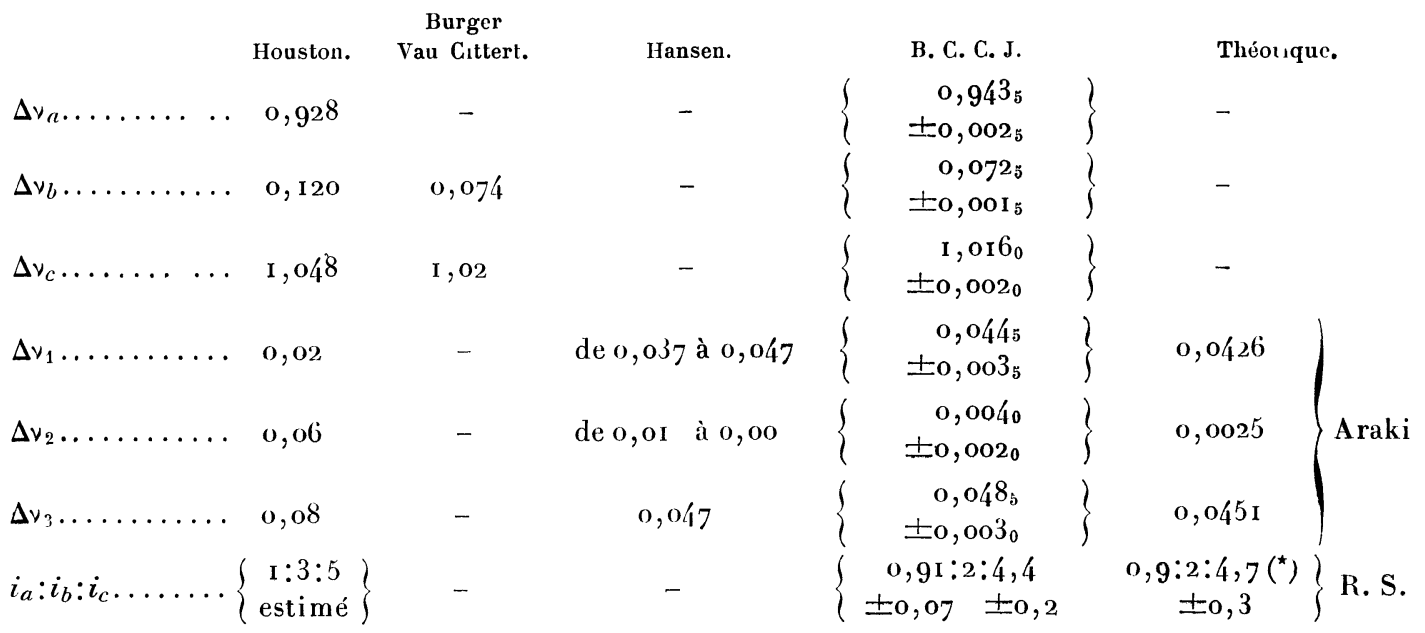

(*) Suivant que l'on tient plus ou moins compte de la composante $2^{3} P_{1}-3^{3} D_{1}$ 
Les résultats de Houston sont probablement dus à ce qu'il observait, en réalité, un doublet dont la composante la plus intense était renversée; en outre, son interprétation de la structure était inexacte, comme l'a montré Grotrian [9], qui a le premier donné une interprétation correcte. Dans un travail de Wei [10], les composantes $b$ et $c$ étaient si loin

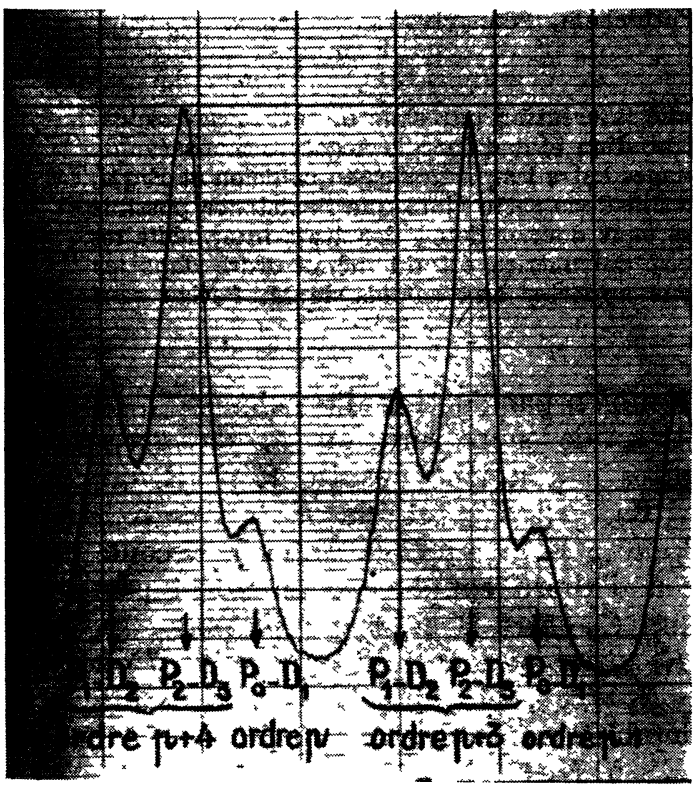

Fig. 8. - Enregistrement de la structure fine de la raie $5875 \AA\left(2^{3} P-3^{3} D\right)$. d'être résolues que son résultat $\left(\Delta \nu_{1}: \Delta \nu_{2}=10,6\right)$ ne peut être considéré que comme un ordre de grandeur.

Burger et Van Cittert [11] ont pris de grandes précautions pour éviter l'auto-absorption : ils ont observé une dissymétrie plus nette que Wei, mais étaient encore loin de résoudre $b$ et $c$. C'est aux mesures de Hansen, faites à la température de l'hydrogène liquide, que l'on doit le plus de crédit. Nos mesures sont en bon accord avec ces dernières : toutefois, comme les écarts des niveaux $D$ ne peuvent s'obtenir que par différence, nos mesures des écarts de composantes étant plus précises que celles de Hansen, la structure du niveau $3^{3} \mathrm{D}$ se trouve nettement mieux connue.

Conclusion. - La structure de trois niveaux de triplets de l'hélium se trouve maintenant bien établie et confirme, dans l'ensemble, les résultats théoriques d'Araki : toutefois, pour le niveau ${ }_{2}^{3} P$, la précision des mesures est telle que l'incertitude $0,001 \mathrm{~cm}^{-1}$ est bien inférieure aux écarts $\left(0,05\right.$ et $\left.0,008 \mathrm{~cm}^{-1}\right)$ entre les valeurs calculées et mesurées, les calculs devront donc être encore perfectionnés pour rendre compte de l'expérience. Quant aux deux autres niveaux étudiés, les valeurs mesurées sont un peu supérieures à celles d'Araki, mais il serait utile d'améliorer encore la précision et les mesures seront reprises prochainement à la température de l'hélium liquide.

\section{Manuscrit reçu le 12 avril 1952.}

BIBLIOGRAPHIE.

[1] Z. Physik, 1926, 39, 349 .

[2] Phys. Rev., I930, 36, 383.

[3] Proc. Phys. math. Soc. Japan, 1937, 19, 128.

[4] Nature (London), 1927, 119, 237 ; Verh. dtch. phys. Ges., $1929,10,5$.

[5] Dufour et JAcQuinot, Journal de Recherches C. N. R. S., 1948, n० 6, p. 9 1.

[6] Proc. Nat. Acad., U. S. A., 1927, 13, 91; Phys, Rev., 1927, 29, 749 .
[7] Phys. Rev., 1931, 37, 1559.

[8] Proc. Roy. Soc., 1951, 209, 327.

[9] Graphische Darstellung der Spektren von Atomen und Ionen mit ein, zwei und drei Valenzelektronen, Berlin, 1928, t. I, p. I 1 3-1 15 .

[10] Astrophys. J., 1926, 68, 246.

[11] Z. Physik, 1928, 51, 638. 